\title{
Investigación sobre el Compromiso o Engagement Académico de los Estudiantes: Una Revisión Sistemática sobre Factores Influyentes y Instrumentos de Evaluación
}

\author{
Researching Academic Engagement in Students: A Systematic Review of the \\ Associated Factors and Assessment Instruments
}

\author{
Begoña María Tortosa Martínez ${ }^{1}$, María del Carmen Pérez-Fuentes² y María del Mar Molero Jurado 3
}

\begin{abstract}
Resumen
El objetivo de este estudio fue identificar y describir los principales instrumentos de medición del compromiso de los estudiantes en el contexto educativo a través del método de revisión sistemática. Se consultaron cuatro bases de datos Scopus, Dialnet, Web of Science y PsycInfo, identificando 15 trabajos para la muestra final. Los resultados indicaron que la etapa donde más se analizó el compromiso académico fue la Educación Secundaria (53.3\%). Se encontraron 11 instrumentos diferentes para la evaluación de este constructo. El instrumento más utilizado fue "Utrecht Work Engagement Scale" de Schaufeli y Bakker (2004), que analiza variables psicológicas, siendo los factores cognitivo, afectivo y compromiso los que producen un interés mayor. Concluyendo, sería aconsejable indagar en otras bases de datos para realizar nuevos análisis de instrumentos que evalúen este constructo. Así como, desarrollar nuevos instrumentos para su evaluación.
\end{abstract}

Palabras clave: compromiso académico, instrumentos, revisión sistemática

\begin{abstract}
This study aimed to identify and describe the main instruments for measuring student engagement in the educational context through the systematic review method. Four databases (Scopus, Dialnet, Web of Science and Psycinfo) were examined and 15 investigations were identified for the final sample. The results indicated that the stage where the academic engagement was most analyzed was secondary education (53.3\%). Eleven different evaluation instruments were found for the evaluation of this construct. The most widely used instrument was the "Utrecht Work Engagement Scale" by Schaufeli y Bakker (2004), which analyzes psychological variables $(100 \%)$, with cognitive factors $(80 \%)$, affective factors $(80 \%)$, and commitment $(80 \%)$ being those that produce a greater interest. In conclusion, it would be advisable to investigate other databases to carry out new analyses of instruments that evaluate this construct. As well as developing new instruments for its evaluation.
\end{abstract}

Keywords: academic engagement, instruments, systematic review

\footnotetext{
${ }^{1}$ Estudiante de doctorado en Educación en la Universidad de Almería. Universidad de Almería. Departamento de Psicología. Universidad de Almería. Ctra. Sacramento s/n, C.P. 04120. La Cañada de San Urbano. Almería, España. Tel.: +34 950015598. Correo: btm172@ual.es

${ }^{2}$ Catedrática de la Universidad de Almería. Universidad de Almería. Departamento de Psicología. Universidad de Almería. Ctra. Sacramento s/n, C.P. 04120. La Cañada de San Urbano. Almería, España. Tel.: +34 950015598. Correo: perezfuentes@ual.es

${ }^{3}$ Profesora Titular de la Universidad de Almería. Universidad de Almería. Departamento de Psicología. Universidad de Almería. Ctra. Sacramento s/n, C.P. 04120. La Cañada de San Urbano. Almería, España. Tel.: +34 950015598. Correo: mmj130@ual.es
} 


\section{Introducción}

El presente artículo surgió a raíz de una revisión documental sobre los factores influyentes e instrumentos de evaluación del compromiso académico de los estudiantes. El progresivo interés en este campo, sobre todo en la última década, ha llevado a que tanto educadores como investigadores estén interesados en la Psicología Educacional Positiva, con el fin de centrarse en aquellos factores que promueven el compromiso hacia las tareas escolares y en desarrollar instrumentos que lo evalúan y midan los diversos componentes que se incluyen en este difícil proceso (Baena-Extremera \& Granero-Gallegos, 2013; D'Mello et al., 2017; Medrano et al., 2014; Pérez-Fuentes et al., 2018). Estos factores engloban todo lo relacionado con la educación, las experiencias académicas y los resultados escolares, tal y como señalan Causo-Holgado et al. (2013), Miranda-Zapata et al. (2018) y Navarro et al. (2014).

Dentro de este modelo, el compromiso escolar ha adquirido un rol fundamental, pues, a nivel conceptual, es considerado por los investigadores y educadores como resultado de una exitosa combinación entre la psicología positiva y la implicación hacia los estudios, teniendo en cuenta las tres dimensiones que lo caracterizan: el vigor, la dedicación y la absorción (Caballero et al., 2015a). Por tanto, es un constructo positivo sobre el aprendizaje que explica las dificultades de motivación, rendimiento y abandono escolar, con el fin de alcanzar el éxito académico (Aspeé et al., 2018; Peña et al., 2017). Además, presenta un vínculo estrecho con numerosos factores sociodemográficos tales como la edad, el sexo y el estado civil (Caballero et al., 2015), y variables propias del ámbito académico como el burnout, el bienestar que presenta el estudiante hacia las metas y retos escolares (Cavazos \& Encinas, 2016; Domínguez et al., 2017; Renshaw \& Long, 2015), la satisfacción y el rendimiento académico, entre otras (Benavente et al., 2018; Guido et al., 2011; Korobova \& Starobin, 2015; Maluenda et al., 2020; Renshaw \& Long, 2015; Tomás et al., 2016). En este sentido, es relevante conocer dichas variables para poder seguir realizando otros estudios sobre el compromiso académico que validen nuevos instrumentos y midan la correlación de unas variables sobre otras (Martos et al., 2018).

Tomando en consideración a autores como Caballero et al. (2015) y Martos et al. (2018), es fundamental conocer las características de este constructo y sus instrumentos de medida aptos para todas las etapas educativas, con el fin de profundizar en el conocimiento del compromiso académico y en su evaluación. Pues el uso de instrumentos no adaptados o validados conlleva a una reducción de la calidad de la investigación y, por ende, de su conocimiento (Lara et al., 2021; Sinatra et al., 2015).

Específicamente, el compromiso académico suele medirse con la escala y factores (dedicación, vigor y absorción) planteados por Schaufeli y Bakker (2004), denominada Utrecht Work Engagement Scale (UWES), existiendo diversos autores que han realizado validaciones $\mathrm{y}$ modificaciones de esta escala (Mills et al., Culbertson \& Fullagar, 2012; Zecca et al., 2015). Al igual, otros estudios han pretendido dar a conocer nuevos instrumentos para medir este constructo, teniendo en cuenta otros factores a analizar (cognitivo, afectivo y conductual) (Lara et al., 2018; Sánchez \& Andrae, 2020). Dadas las características y diferencias de las revisiones previas, el artículo aporta una revisión vigente sobre los instrumentos que se utilizan para medir el compromiso académico de los estudiantes, con el fin de que se considere el estudio de aspectos metodológicos para avanzar en intervenciones e investigaciones en este campo. Desde esta perspectiva, el objetivo principal de esta revisión sistemática es identificar y describir los principales instrumentos de medición del compromiso de los estudiantes, en el contexto educativo.

\section{Metodología}

\section{Procedimiento}

Para lograr el objetivo planteado se siguió la metodología de revisión sistemática basada en las recomendaciones de la Declaración PRISMA (Moher et al., 2015), la cual se desarrolló en dos fases. La primera consistió en identificar los estudios a incluir y la segunda, en extraer la información de los estudios para analizarlos posteriormente. El processo de selección de los 


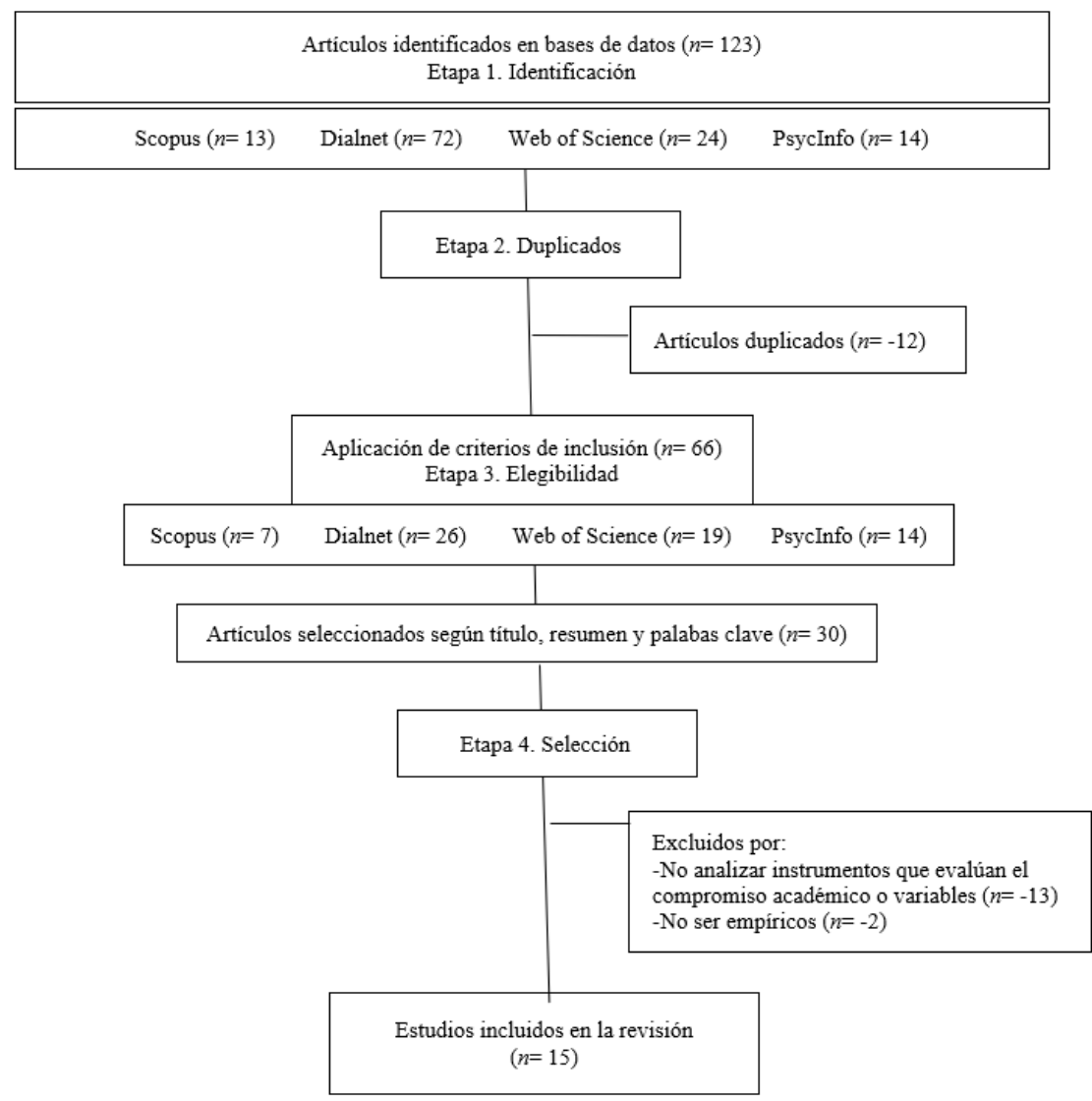

Figura 1. Diagrama de flujo con el proceso de selección de documentos

Tabla 1. Bases de datos, descriptores, fórmulas de búsqueda y $\mathrm{n}^{\circ}$ de referencias

\begin{tabular}{|c|c|c|c|}
\hline Base de datos & Descriptores & Fórmulas de búsqueda & $\mathrm{N}^{\circ}$ Referencias \\
\hline Scopus & $\begin{array}{c}\text { "Academic engagement and } \\
\text { instrument and validation and } \\
\text { adolescent" }\end{array}$ & $\begin{array}{l}\text { ((Academic engagement) AND (instrument) AND (validation) } \\
\text { AND (adolescent) }) \text {. } \\
\text { ((“compromiso académico" OR “academic engagement”) AND } \\
\text { (instrument) AND (validation) AND (adolescent OR students)). }\end{array}$ & 8 \\
\hline Dialnet & $\begin{array}{l}\text { "Compromiso académico and } \\
\text { instrumento and validación and } \\
\text { adolescentes" }\end{array}$ & $\begin{array}{l}\text { ((Compromiso-académico) AND (instrumento) AND (validación) } \\
\text { AND (adolescentes)) } \\
\text { ((“Compromiso académico” OR engagement) AND instrumento } \\
\text { AND validación AND (adolescentes OR estudiantes)). }\end{array}$ & 41 \\
\hline $\begin{array}{l}\text { Web of } \\
\text { Science }\end{array}$ & $\begin{array}{c}\text { "Academic engagement and } \\
\text { instrument and validation and } \\
\text { adolescent" }\end{array}$ & $\begin{array}{l}\text { ((Academic engagement) AND (instrument) AND (validation) } \\
\text { AND (adolescent })) \text {. } \\
\text { ((“Compromiso académico” OR engagement) AND instrumento } \\
\text { AND validación AND (adolescentes OR estudiantes)). }\end{array}$ & 17 \\
\hline PsycInfo & $\begin{array}{c}\text { "Academic engagement and } \\
\text { instrument and validation and } \\
\text { adolescent" }\end{array}$ & $\begin{array}{l}\text { ((Academic engagement) AND (instrument) AND (validation) } \\
\text { AND (adolescent })) \\
\text { ((“compromiso académico" OR “academic engagement”) AND } \\
\text { (instrument) AND (validation) AND (adolescent OR students)). }\end{array}$ & 9 \\
\hline
\end{tabular}

artículos conllevó 4 etapas (identificación, duplicado, elegibilidad y selección) que se pueden visualizar en la Figura 1.

En la etapa de identificación, se realizó una búsqueda exhaustiva de publicaciones en las siguientes bases de datos, todas ellas relacionadas con las ciencias sociales, de la educación, de la salud y psicología: Scopus, Dialnet, Web of Science y PsycInfo. Como descriptores se usó, en español y en inglés, el término base "compromiso académico". Así como "instrumento", "validación" y “adolescentes" en español y en inglés y se usaron los operadores booleanos de proximidad “AND" y "OR". El descriptor "compromiso académico" se aplicó al título, resumen y palabras clave de los estudios, siendo esencial que apareciese junto con algunos de los descriptores secundarios. Las fórmulas de búsqueda fueron: ((compromiso académico) AND (instrumento) AND (validación) AND (adolescentes)), ((“Compromiso académico" OR engagement) AND instrumento AND validación AND 
Tabla 2. Características generales de los estudios seleccionados

\begin{tabular}{|c|c|c|c|c|c|c|c|}
\hline Autor (año) & País & Instrumento & $\begin{array}{c}\text { Muestra a la que } \\
\text { se dirige (edad) }\end{array}$ & $\mathrm{N}^{o}$ ítems y formato & Dimensiones & $\begin{array}{c}\text { Escalas y } \\
\text { subescalas }\end{array}$ & Fiabilidad estimada \\
\hline Whitney et al. (2019) & EE.UU. & $\begin{array}{c}\text { Scale of Student Engagement } \\
\text { in Statistics (SSE-S, Whitney } \\
\text { et al., 2019) }\end{array}$ & $\begin{array}{l}195 \text { adolescentes } \\
(14-18)\end{array}$ & $\begin{array}{c}24 \\
\text { Likert con } 5 \text { puntos }\end{array}$ & 3 & $\begin{array}{l}\text {-Cognitivo. } \\
\text {-Afectivo. } \\
\text {-Conductual. }\end{array}$ & $\begin{array}{c}\alpha=.79 \text { (cognitiva) } \\
\alpha=.89 \text { (afectiva) } \\
\alpha=.85 \text { (conductual) } \\
\alpha=.88 \text { (total) }\end{array}$ \\
\hline Luo et al. (2019) & $\begin{array}{l}\text { China y } \\
\text { España }\end{array}$ & $\begin{array}{l}\text { Academic Interest Scale for } \\
\text { Adolescents (AISA, Luo, } \\
\text { Dang, y Xu, 2019) }\end{array}$ & $\begin{array}{c}3139 \\
\text { adolescentes } \\
(10-15)\end{array}$ & $\begin{array}{c}29 \\
\text { Likert con } 5 \text { puntos }\end{array}$ & 4 & $\begin{array}{l}\text {-Emoción. } \\
\text {-Valor. } \\
\text {-Conocimiento. } \\
\text {-Compromiso. }\end{array}$ & $\begin{array}{c}\alpha=.90 \text { (emoción) } \\
\alpha=.93 \text { (valor) } \\
\alpha=.93 \text { (conocimiento) } \\
\alpha=.91 \text { (compromiso) en } \\
\text { inglés }\end{array}$ \\
\hline Lara et al. (2018) & Chile & $\begin{array}{c}\text { Cuestionario del compromiso } \\
\text { de los adolescentes (Lara et } \\
\text { al., 2018) }\end{array}$ & $\begin{array}{l}454 \text { adolescentes } \\
(10-16)\end{array}$ & $\begin{array}{c}57 \\
\text { Likert con } 5 \text { puntos }\end{array}$ & 3 & $\begin{array}{l}\text {-Afectiva. } \\
\text {-Conductual. } \\
\text {-Cognitiva. }\end{array}$ & $\begin{array}{c}\alpha=.83 \text { (afectiva) } \\
\alpha=.87 \text { (cognitiva) } \\
\alpha=.86 \text { (conductual) } \\
\alpha=.95 \text { (total) }\end{array}$ \\
\hline $\begin{array}{l}\text { Sánchez y Andrae } \\
\text { (2020) }\end{array}$ & México & $\begin{array}{c}\text { Escala de Involucramiento } \\
\text { Escolar para Adolescentes } \\
\text { (EIE-A, Sánchez y Andrae, } \\
2020)\end{array}$ & $\begin{array}{l}503 \text { adolescentes } \\
(10-15)\end{array}$ & $\begin{array}{c}10 \\
\text { Likert con } 7 \text { puntos }\end{array}$ & 3 & $\begin{array}{l}\text {-Emocional. } \\
\text {-Conductual. } \\
\text {-Cognitiva. }\end{array}$ & $\begin{array}{c}\alpha=.83 \text { (afectiva) } \\
\alpha=.86 \text { (conductual) } \\
\alpha=.74 \text { (cognitiva) } \\
\alpha=.88 \text { (total) }\end{array}$ \\
\hline Appleton et al. (2016) & EE.UU. & $\begin{array}{c}\text { Student Engagement } \\
\text { Instrument (SEI, Appleton y } \\
\text { Christenson, 2004) }\end{array}$ & $\begin{array}{c}2577 \\
\text { adolescentes } \\
(14-15)\end{array}$ & $\begin{array}{c}56 \\
\text { Likert con } 4 \text { puntos }\end{array}$ & 4 & $\begin{array}{l}\text {-Académica. } \\
\text {-Comportamental. } \\
\text {-Cognitiva. } \\
\text {-Psicológica. }\end{array}$ & $\alpha=.72$ (total) \\
\hline Bae Han (2019) & EE.UU. & $\begin{array}{c}\text { Academic Engagement and } \\
\text { Self-reported Learning } \\
\text { Abilities (SRLA, Bae y Han, } \\
\text { 2019) }\end{array}$ & $\begin{array}{l}42260 \text { estudiantes } \\
\text { universitarios } \\
(-)\end{array}$ & $\begin{array}{c}37 \\
\text { Likert con } 6 \text { puntos } \\
\text { (engagement) y } 8 \\
\text { puntos (learning) }\end{array}$ & $\begin{array}{c}5 \\
\text { (engagement) } \\
3 \\
\text { (learning) }\end{array}$ & $\begin{array}{l}\text {-Atención e interés } \\
\text { en clase. } \\
\text {-Preparación de la } \\
\text { clase. } \\
\text {-Tiempo impartido. } \\
\text {-Aprendizaje } \\
\text { consolidado y por } \\
\text { pares. } \\
\text {-Interacción con el } \\
\text { centro. } \\
\text {-Habilidades de } \\
\text { conocimiento y } \\
\text { comprensión. } \\
\text {-Habilidades de } \\
\text { búsqueda. } \\
\text {-Habilidades de } \\
\text { comunicación. }\end{array}$ & $\begin{array}{l}\alpha=>.73 \text { (engagement) } \\
\alpha=.75 \text { a } .82 \text { (learning) }\end{array}$ \\
\hline $\begin{array}{l}\text { Ramos-Díaz et al. } \\
\text { (2016) }\end{array}$ & España & $\begin{array}{l}\text { Versión en español del The } \\
\text { School Engagememt Measure } \\
\text { (Frederiks, Blumenfeld, } \\
\text { Friedel, y Paris, 2005) } \\
\text { validado por Ramos-Díaz y } \\
\text { Rodríguez-Fernández (2016) }\end{array}$ & $\begin{array}{l}1250 \\
\text { adolescentes (12- } \\
15)\end{array}$ & $\begin{array}{c}19 \\
\text { Likert de } 5 \text { puntos }\end{array}$ & 3 & $\begin{array}{l}\text {-Compromiso } \\
\text { comportamental. } \\
\text {-Compromiso } \\
\text { emocional. } \\
\text {-Compromiso } \\
\text { cognitivo. }\end{array}$ & $\begin{array}{c}\alpha=.74 \text { (conductual) } \\
\alpha=.81 \text { (afectiva) } \\
\alpha=.77 \text { (cognitiva) }\end{array}$ \\
\hline Veiga (2012) & Portugal & $\begin{array}{l}\text { The Students' Engagement } \\
\text { Scale (Veiga, 2012) }\end{array}$ & $\begin{array}{l}217 \text { estudiantes } \\
\quad(12-15)\end{array}$ & $\begin{array}{c}20 \\
\text { Likert de } 4 \text { puntos }\end{array}$ & 2 & $\begin{array}{l}\text {-Cognitiva. } \\
\text {-Afectiva. }\end{array}$ & $\begin{array}{c}\alpha=.83 \\
\text { (cognitiva) } \\
\alpha=.45 \\
\text { (afectiva) }\end{array}$ \\
\hline $\begin{array}{l}\text { Chun-tat y Siu-man } \\
\text { (2012) }\end{array}$ & China & $\begin{array}{c}\text { Versión en chino The Utrecht } \\
\text { Work Engagement } \\
\text { Questionnaire (UWES-17, } \\
\text { Schaufeli et al., 2002; } \\
\text { UWES-9, Schaufeli y } \\
\text { Bakker, 2004) validado por } \\
\text { Chun-tat y Siu-man (2012) }\end{array}$ & $\begin{array}{c}992 \text { trabajadores } \\
(18-62)\end{array}$ & $\begin{array}{c}\text { UWES-17 } \\
17 \\
\text { UWES-9 } \\
9 \\
\text { Likert de } 6 \text { puntos }\end{array}$ & 3 & $\begin{array}{l}\text {-Vigor. } \\
\text {-Dedicación. } \\
\text {-Absorción. }\end{array}$ & $\begin{array}{c}\text { UWES- } 9 \\
\alpha=.74 \text { (vigor) } \\
\alpha=.77 \text { (dedicación) } \\
\alpha=.70 \text { (absorción) } \\
\alpha=.88 \text { (engagement) }\end{array}$ \\
\hline
\end{tabular}

Nota. $\alpha=$ coeficiente alfa.

(adolescentes OR estudiantes)), ((academic engagement) AND (instrument) AND (validation) AND (adolescent)) y ((“compromisso académico" OR "academic engagement") AND (instrument) AND (validation) AND (adolescent OR students)) (Tabla 1).

\section{Criterios de elegibilidad}

Para la selección de trabajos se tuvieron en cuenta una serie de criterios de inclusión: (a) que el objetivo del estudio se centrara en el análisis de los instrumentos que evalúan el compromiso académico de los estudiantes. Estudios relacionados con otros tipos de compromisos que no sean con los estudios fueron excluidos, (b) estudios empíricos provenientes de revistas científicas especializadas. Documentos publicados en páginas web, actas de congreso, tesis doctorales, capítulos y notas editoriales. Así como estudios reflexivos y teóricos fueron excluidos, (c) idioma de publicación: español o inglés, excluyendo aquellos estudios en otros idiomas, a pesar de que el resumen estuviera escrito en español o inglés, (d) textos completos. Se excluyeron aquellos a los que no se podía acceder al texto completo. 
Tabla 2. Características generales de los estudios seleccionados (cont.)

\begin{tabular}{|c|c|c|c|c|c|c|c|}
\hline Autor (año) & País & Instrumento & $\begin{array}{l}\text { Muestra a la que } \\
\text { se dirige (edad) }\end{array}$ & $\begin{array}{c}\mathrm{N}^{\circ} \text { ítems } \mathrm{y} \\
\text { formato }\end{array}$ & Dimensiones & Escalas y subescalas & Fiabilidad estimada \\
\hline $\begin{array}{l}\text { Zecca et al. } \\
\text { (2015) }\end{array}$ & Francia & $\begin{array}{c}\text { Versión en francés The Utrecht } \\
\text { Work Engagement Questionnaire } \\
\text { (UWES, Schaufeli y Bakker, } \\
\text { 2004) validado por Zecca et al. } \\
\text { (2015) }\end{array}$ & $\begin{array}{c}661 \text { trabajadores } \\
(19-64)\end{array}$ & $\begin{array}{l}\text { UWES-17 } \\
17 \\
\text { UWES-9 } \\
9 \\
\text { Likert de } 6 \\
\text { puntos }\end{array}$ & 3 & $\begin{array}{l}\text {-Vigor. } \\
\text {-Dedicación. } \\
\text {-Absorción. }\end{array}$ & $\begin{array}{l}\alpha=.<70 \\
\text { (total) }\end{array}$ \\
\hline $\begin{array}{l}\text { Mills et al. } \\
\text { (2012) }\end{array}$ & EE.UU. & $\begin{array}{c}\text { The Utrecht Work Engagement } \\
\text { Questionnaire (UWES-17, } \\
\text { Schaufeli et al., 2002; UWES-9, } \\
\text { Schaufeli y Bakker, 2004) } \\
\text { validado por Mills et al. (2012) }\end{array}$ & $\begin{array}{c}344 \\
\text { universitarios } \\
\text { (edad media de } \\
21.32 \text { años) }\end{array}$ & $\begin{array}{l}\text { UWES-17 } \\
17 \\
\text { UWES-9 } \\
9 \\
\text { Likert de } 6 \\
\text { puntos }\end{array}$ & 3 & $\begin{array}{l}\text {-Vigor. } \\
\text {-Dedicación. } \\
\text {-Absorción. }\end{array}$ & $\begin{array}{c}\text { UWES-17 } \\
\alpha=.90 \text { (vigor) } \\
\alpha=.86 \text { (dedicación) } \\
\alpha=.86 \text { (absorción) } \\
\alpha=.95 \text { (engagement) } \\
\text { UWES-9 } \\
\alpha=.82 \text { (vigor) } \\
\alpha=.83 \text { (dedicación) } \\
\alpha=.70 \text { (absorción) } \\
\alpha=.91 \text { (engagement) }\end{array}$ \\
\hline $\begin{array}{l}\text { Soane et al. } \\
\text { (2012) }\end{array}$ & Reino Unido & $\begin{array}{l}\text { The Intellectual, Social and } \\
\text { Affective Engagement Scale } \\
\text { (ISA, Soane et al., 2012) }\end{array}$ & $\begin{array}{l}540 \text { trabajadores } \\
\text { (edad media de } \\
39.88 \text { años) }\end{array}$ & $\begin{array}{c}17 \\
\text { Likert de } 7 \\
\text { puntos }\end{array}$ & 3 & $\begin{array}{l}\text {-Física. } \\
\text {-Cognitiva. } \\
\text {-Emocional. }\end{array}$ & $\begin{array}{c}\alpha=.88 \text { (física) } \\
\alpha=.95 \text { (emocional) } \\
\alpha=.95 \text { (cognitiva) } \\
\alpha=.88 \text { (engagement) }\end{array}$ \\
\hline $\begin{array}{l}\text { Mañas- } \\
\text { Rodríguez et } \\
\text { al. (2016) }\end{array}$ & España & $\begin{array}{l}\text { Spanish Version of ISA } \\
\text { Engagement Scale (ISA, Soane et } \\
\text { al., 2012) validado por Mañas- } \\
\text { Rodríguez et al. (2016) }\end{array}$ & $\begin{array}{c}477 \\
\text { universitarios } \\
(36-55)\end{array}$ & $\begin{array}{c}9 \\
\text { Likert de } 7 \\
\text { puntos }\end{array}$ & 3 & $\begin{array}{l}\text {-Social. } \\
\text {-Intelectual. } \\
\text {-Afectivo. }\end{array}$ & $\begin{array}{c}\alpha=.92 \text { (social) } \\
\alpha=.94 \text { (emocional) } \\
\alpha=.90 \text { (intelectual) } \\
\alpha=.91 \text { (engagement) } \\
\alpha=.76 \text { (cognitiva) }\end{array}$ \\
\hline Veiga (2016) & Portugal & $\begin{array}{c}\text { Students' Engagement in School } \\
\text { Four-Dimensional Scale (SES- } \\
\text { 4DS, Veiga, 2013) }\end{array}$ & $\begin{array}{c}685 \text { adolescentes } \\
(11-16)\end{array}$ & $\begin{array}{l}20 \\
\text { Likert de } 6 \\
\text { puntos }\end{array}$ & 4 & $\begin{array}{l}\text {-Cognitiva. } \\
\text {-Afectiva. } \\
\text {-Comportamental. } \\
\text {-Agéntica. }\end{array}$ & $\begin{array}{c}\alpha=.82 \text { (afcetiva) } \\
\alpha=.70 \text { (comportamental) } \\
\alpha=.85 \text { (agéntica) } \\
\alpha=.82 \text { (total) }\end{array}$ \\
\hline $\begin{array}{l}\text { LaNasa et al. } \\
\text { (2010) }\end{array}$ & EE.UU. & $\begin{array}{c}\text { National Survey of Student } \\
\text { Engagement (NSSE, Kuh, 2000) } \\
\text { validado por LaNasa et al. (2009) }\end{array}$ & $\begin{array}{l}375 \\
\text { universitarios (-) }\end{array}$ & 37 & 8 & $\begin{array}{c}\text {-Estrategias de } \\
\text { aprendizaje. } \\
\text {-Interacción } \\
\text { académica. } \\
\text {-Empatía. } \\
\text {-Actividades co- } \\
\text { curriculares. } \\
\text {-Interacciones } \\
\text { diversas. } \\
\text {-Esfuerzo. } \\
\text {-Relaciones. } \\
\text {-Carga de trabajo. }\end{array}$ & $\begin{array}{c}\alpha=.84 \text { (Estrategias) } \\
\alpha=.75 \text { (Interacción } \\
\text { académica) } \\
\alpha=.81 \text { (Empatía) } \\
\alpha=.58 \text { (Actividades) } \\
\alpha=.88 \text { (Interacciones) } \\
\alpha=.52 \text { (Esfuerzo) } \\
\alpha=.70 \text { (Relaciones) } \\
\alpha=.58 \text { (Carga) }\end{array}$ \\
\hline
\end{tabular}

\section{Selección de documentos}

La Figura 1 presenta el proceso de selección de estudios (diagrama de flujo). Se identificaron un total de 123 trabajos tras la búsqueda inicial. Los documentos se dividieron de la siguiente manera: Scopus (13), Dialnet (72), Web of Science (24) y PsycInfo (14). La mayor parte de los artículos se encontraron en la base de datos de Dialnet, resultando la duplicación de artículos en las bases de datos de Scopus, Web of Science y PsycInfo. Hubo 12 artículos duplicados.

A continuación, siguiendo lo explicitado en los subapartados de procedimiento y criterios de elegibilidad, se aplicaron los filtros y los criterios de inclusión, seleccionando 66 estudios para su clasificación manual. Tras la lectura del título, resumen y texto completo de los artículos, se aplicaron los criterios de exclusión mencionados, quedando finalmente 15 artículos que cumplían los criterios de selección y resultaban interesantes para el objetivo de esta revisión, quedando Scopus (2), Dialnet (3), Web of Science (2) y PsycInfo (3).

\section{Resultados}

Los resultados se exponen considerando 4 aspectos centrales analizados en los estudios seleccionados: 1) países donde se desarrollaron los estudios; 2) nombre de los instrumentos usados para medir el compromiso académico; 3) agrupamiento de variables y factores analizados; 4) año de publicación, idioma y nivel educativo al que van dirigidos.

\section{Países donde se desarrollaron los estudios}

Del total de 15 trabajos, los estudios con más frecuencia se distribuyen por el continente de América del Norte, comprendiendo el país de Estados Unidos (EE.UU.) (33.3\%). El segundo país con más investigaciones en este constructo es España (20\%), seguido de Portugal y China (13.3\%). La menor frecuencia de estudios realizados fue en Chile, México, Francia y Reino Unido (6.7\%). Cabe destacar que todos los estudios recogieron pruebas en un solo país. 
Excepto uno de ellos, la investigación de Luo, Dang, y Xu (2019) la cual recogió datos en dos países (España y China) (Tabla 2).

\section{Instrumentos utilizados para medir el compromiso académico}

Tal y como se contempla en la Tabla 3, el instrumento más utilizado para medir el compromiso académico de los estudiantes ha sido el UWES "The Utrecht Work Engagement Questionnaire" de Schaufeli y Bakker (2004) (20\%), prestando atención a que cada estudio ha validado versiones traducidas a diversos idiomas como el español, el francés o el chino. Posteriormente, se observa que los autores tienen una tendencia a usar la ISA "The Intellectual, Social and Affective Engagement Scale" de Soane et al. (2012) (13.3\%). Por el contrario, algunos de los instrumentos que se suelen usar con menos frecuencia han sido el "Cuestionario del compromiso de los adolescentes" de Lara et al. (2018), la "Students' Engagement in School FourDimensional Scale" de Veiga (2013) y el "Student Engagement Instrument" de Appleton et al. (2016), entre otros, teniendo una prevalencia de 6.7\% cada uno de ellos. En total se encontraron 11 instrumentos que permiten evaluar el compromiso académico de los estudiantes.

Tabla 3. Instrumentos usados en la medición del

\begin{tabular}{lc}
\multicolumn{2}{c}{ engagement académico } \\
\hline Instrumentos & $\%$ de artículos \\
\hline 1. SSE-S & $6.7 \%(n=1)$ \\
2. AISA & $6.7 \%(n=1)$ \\
3. Cuestionario del compromiso de los & $6.7 \%(n=1)$ \\
adolescentes & $6.7 \%(n=1)$ \\
4. EIE-A & $6.7 \%(n=1)$ \\
5. SEI & $6.7 \%(n=1)$ \\
6. SRLA & $6.7 \%(n=1)$ \\
7. The School Engagememt Measure & $13.3 \%(n=2)$ \\
8. SES & $20 \%(n=3)$ \\
9. UWES & $13.3 \%(n=2)$ \\
10. ISA & $6.7 \%(n=1)$ \\
11. NSSE &
\end{tabular}

\section{Agrupamiento de variables y factores analizados}

Las variables analizadas por los autores de los artículos estudiados se dividieron, según su naturaleza, en 5 grupos, encontrando un total de 24 factores distribuidos en las variables de índole sociodemográficas, contextuales, académicas, psicológicas y sociales, tal y como se expone en la Tabla 4
Tabla 4. Agrupamiento de variables, factores analizados y su uso en los artículos

\begin{tabular}{|c|c|c|}
\hline Variables & $\begin{array}{c}\mathrm{N}^{\circ} \text { de } \\
\text { artículos }\end{array}$ & Factores más mencionados \\
\hline Sociodemográficas & $\begin{array}{c}33.3 \% \\
(n=5)\end{array}$ & $\begin{array}{c}\text { Sexo y edad: } 26.7 \%(n=4) \\
\text { Raza: } 6.7 \%(n=1)\end{array}$ \\
\hline Contextuales & $\begin{array}{l}20 \% \\
(n=3)\end{array}$ & $\begin{array}{l}\text { Clima escolar: } 6.7 \%(n=1) \\
\text { Educación parental: } 6.7 \%(n=1)\end{array}$ \\
\hline Académicas & $\begin{array}{l}33.3 \% \\
(n=5)\end{array}$ & $\begin{array}{c}\text { Nivel educativo: } 26.7 \%(n=4) \\
\text { Rendimiento: } 20 \%(n=3) \\
\text { Satisfacción académica: } 13 \%(n=2) \\
\text { Calificaciones: } 13 \%(n=2)\end{array}$ \\
\hline & & $\begin{array}{c}\text { Cognitivo: } 46.7 \%(n=7) \\
\text { Afectivo: } 46.7 \%(n=7) \\
\text { Comportamental: } 46.7 \%(n=7) \\
\text { Dimensión organizacional: } 6.7 \%(n=1) \\
\text { Dimensión agéntica: } 6.7 \%(n=1)\end{array}$ \\
\hline Psicológicas & $\begin{array}{l}100 \% \\
(n=15)\end{array}$ & $\begin{array}{c}\text { Compromiso: } 53.3 \%(n=8) \\
\text { Vigor: } 40 \%(n=6) \\
\text { Dedicación: } 40 \%(n=6) \\
\text { Absorción: } 40 \%(n=6) \\
\text { Bienestar: } 6.7 \%(n=1) \\
\text { Motivación: } 6.7 \%(n=1) \\
\text { Autoeficacia: } 6.7 \%(n=1) \\
\text { Confianza: } 6.7 \%(n=1)\end{array}$ \\
\hline Sociales & $\begin{array}{l}20 \% \\
(n=3)\end{array}$ & $\begin{array}{c}\text { Apoyo social: } 13.3 \%(n=2) \\
\text { Relaciones sociales: } 6.7 \%(n=1)\end{array}$ \\
\hline
\end{tabular}

En relación al número de variables mencionadas en las publicaciones (Tabla 4), se contempla que las psicológicas son las más mencionadas (100\%), seguidas de las académicas (33.3\%), sociodemográficas (33.3\%), contextuales y sociales $(20 \%)$. Dentro de la primera categoría, los factores que más se han analizado han sido el "cognitivo" (80\%), "afectivo" (80\%), "comportamental" (60\%), el "compromiso" (80\%), el "vigor" (60\%), la "dedicación" $(50 \%)$ y la "absorción" (50\%). Por otra parte, otra variable frecuentemente mencionada ha sido la académica, analizando en mayor medida el factor de "nivel educativo" (57.1\%).

Un resultado adicional que podemos observar al analizar la Tabla 4, es el análisis de los factores o dimensiones que agregan otros instrumentos y que no son considerados en UWES. Concretamente, el instrumento con mayor frecuencia de uso correspondiente al UWES, considera las dimensiones de: vigor, dedicación y absorción.

En el caso de los 10 instrumentos restantes, es importante la aparición de otras dimensiones tenidas en cuenta por los autores, en las que se observa que la conceptualización usada es igual que algunas dimensiones del UWES. Esto se puede observar en Veiga (2016), Whitney et al. (2019), Lara et al. (2018), Sánchez y Andrae (2020), Appleton et al. (2016) y Ramos-Díaz y 
Rodríguez-Fernández (2016), quienes refieren la dimensión cognitiva como equivalente al vigor del estudiante a la hora de comprometerse, la dimensión afectiva como equivalente a la dedicación y la dimensión comportamental o conductual correspondiente a la absorción.

Respecto a la equivalencia de las dimensiones, también se encuentran algunas inconsistencias: por ejemplo, algunos autores como Bae y Han (2019), Soane et al. (2012), Mañas-Rodríguez et al. (2016), Veiga (2016) y LaNasa et al. (2010), para evaluar el compromiso de los estudiantes, tienen en cuenta factores como el tiempo, la interacción social, una serie de habilidades de comprensión, búsqueda y comunicación; estrategias de aprendizaje, la carga de trabajo y el factor físico y social. Por ello, se proponen futuras investigaciones centradas en el desarrollo de instrumentos para medir el compromiso académico, considerando las diferentes variables y dimensiones implicadas en los estudios analizados.

\section{Año de publicación, idioma y nivel educativo}

Los años en los que ha habido más publicaciones en relación a los instrumentos del compromiso académico han sido el $2012(n=4)$, acompañado del $2016(n=3)$ y $2019(n=3)$. El 86. $7 \%$ de los artículos están redactados en inglés, mientras que el 13.3\% están escritos en español.

En cuanto al nivel educativo elegido por los autores, sólo dos de los trabajos han tenido en cuenta dos etapas educativas. Pero, la etapa en la que predomina más el estudio de este constructo es en la Educación Secundaria, siendo el 53.3\% de los trabajos analizados y la etapa universitaria llega a un $46.7 \%$ de los estudios, siendo la Educación Primaria la menos estudiada (13.3\%).

\section{Discusión/Conclusiones}

El objetivo de este estudio fue identificar y describir los principales instrumentos de medición del compromiso de los estudiantes, en el contexto educativo. Pues, en la última década, se presta atención a la importancia de su investigación, sobre todo en EE.UU. y España, aportándole valor a factores académicos, psicológicos, personales y sociales (Caballero et al., 2015b). Para dar respuesta al objetivo planteado, se realizó una revisión sistemática de artículos cuantitativos, publicados recientemente en revistas indexadas en las bases de datos de Scopus, Dialnet, Web of Science y PsycInfo. En cuanto al análisis de los países donde más investigaciones se han desarrollado, se halló que Estados Unidos concentra el mayor número de estudios relacionados con el compromiso en el contexto educativo, contribuyendo pues al desarrollo y adaptaciones de instrumentos para evaluar dicho constructo. Este resultado es concordante con los hallazgos de Lara et al. (2021) y Sinatra et al. (2015), quienes identificaron que la mayoría de instrumentos para la medida del compromiso se han creado en países anglosajones. Esto puede deberse a que el compromiso académico comenzó a ser estudiado en estos países, en la década de 1970 (Aspeé et al., 2018), bajo el nombre de “involucramiento estudiantil" (Peña et al., 2017).

Respecto a los instrumentos hallados para medir el compromiso académico de los estudiantes, se encontraron específicamente 11 instrumentos, siendo el UWES de Schaufeli y Bakker (2004) el más utilizado. Este instrumento evalúa las dimensiones de dedicación, vigor y absorción y está validado en múltiples contextos, considerándose el más eficaz y fiable en el ámbito educativo (Mills et al., 2012). Además, a pesar de que se encuentran otros instrumentos que se centran en la medición de variables académicas y/o contextuales, el instrumento más usado es aquel que evalúa el compromiso de los estudiantes gracias a variables psicológicas (Chun-tat \& Siuman, 2012; Mills et al., 2012; Zecca et al., 2015). En este sentido, no existe consenso sobre qué instrumento es el más adecuado, pues existen deficiencias en cuanto a la adecuación de las propiedades psicométricas y confusión sobre las variables con las que el constructo se relaciona (Sinatra et al., 2015). De aquí la necesidad de contar con revisiones de instrumentos que evalúen el compromiso académico, para visualizarlos y fomentar estrategias para su desarrollo en estudiantes (Lara et al., 2021; Sinatra et al., 2015). Así como, conocer las características e instrumentos de medida del compromiso escolar y de aquellas variables que lo promueven, con la finalidad de mejorar el aprendizaje y bienestar académico de los alumnos y alumnas (BaenaExtremera \& Granero-Gallegos, 2013; Caballero 
et al., 2015 a ; Martos et al., 2018; Medrano et al., 2014).

Entre las limitaciones de este trabajo se encuentra la omisión de estudios, de manera involuntaria, publicados en otras bases de datos. Puesto que la estrategia de búsqueda se ha limitado en las bases de datos de Scopus, Dialnet, Web of Science y PsycInfo, lo que puede dificultar en los hallazgos de un número mayor de investigaciones cuantitativas relacionadas a los instrumentos de medida del compromiso de los estudiantes en el contexto escolar. La inclusión de otras bases de datos diferentes favorecería la incorporación de otros trabajos. Así como el uso exclusivo de publicaciones con acceso al texto completo, con la finalidad de evitar pérdidas de información al analizarlas en profundidad. En consecuencia, se proponen futuras investigaciones centradas en el desarrollo de instrumentos para medir el compromiso académico, considerando las diferentes variables y dimensiones implicadas en los estudios analizados. Además, sería aconsejable realizar nuevos estudios bibliométricos, teniendo en cuenta otras bases de datos, otras revistas científicas externas a estas bases de datos, otros países y variables, etc., con la intención de ampliar los conocimientos sobre el tema $y$ entender este constructo y su medición de una forma más profunda.

La pertenencia de la utilización de instrumentos de evaluación confiables y válidos; al igual que el desarrollo de estrategias que fomenten el compromiso académico de los estudiantes, como una herramienta para prevenir y reducir la deserción y el fracaso escolar, contribuirá al bienestar de los mismos. Se requiere avanzar en nuevas formas de medición del compromisso académico para evaluar adecuadamente el impacto de programas ya diseñados e implementados con el fin de aumentar los niveles de compromiso académico de los estudiantes.

\section{Agradecimientos}

Este trabajo cuenta con el apoyo del Ministerio de Educación y Formación Profesional a través del programa de Ayudas para la Formación del Profesorado Universitario (FPU) otorgado a Begoña María Tortosa Martínez con referencia FPU19/01568.

\section{Referencias}

Appleton, J. J., Christenson, S. L., Kim, D., \& Reschly, A. L. (2016). Measuring cognitive and psychological engagement: Validation of the Student Engagement Instrument, Journal of School Psychology, 44(5), 427-445. https://doi.org/10.1016/j.jsp.2006.04.002

Aspeé, J., González, J., \& Cavieres-Fernández, E. (2018). Compromiso estudiantil en educación superior como agencia compleja. Formación Universitaria, 11(4), 95-108.

https://doi.org/10.4067/S071850062018000400095

Bae, Y., \& Han, S. (2019). Academic engagement and learning outcomes of the student experience in the research university: Construct validation of the instrument. Educational Sciences: Theory and Practice, 19(3), 49-64.

https://doi.org/10.12738/estp.2019.3.004

Baena-Extremera, A., \& Granero-Gallegos, A. (2013). Efecto de un programa de Educación de Aventura en la orientación al aprendizaje, satisfacción y auto-concepto en secundaria. Revista Iberoamericana de Diagnóstico y Evaluación - e Avaliação Psicológica, 36(2), 163-187.

Benavente, M., Cova, F., Pérez-Salas, C.P., Varela, J. J., Alfaro, J., \& Chuecas, J. (2018). Propiedades psicométricas de la Escala Breve de Bienestar Subjetivo en la Escuela para Adolescentes (BASWBSS) en una muestra de adolescentes chilenos. Revista Iberoamericana de Diagnóstico y Evaluación - e Avaliação Psicológica, 48(3), 53-65. https://doi.org/10.21865/RIDEP48.3.05

Caballero, C.C., Bresó, E., \& González-Gutiérrez, O. (2015a). Burnout en estudiantes universitarios. Psicología desde el Caribe, 32(3), 425-441.

https://doi.org/10.14482/psdc.32.3.6217

Caballero, C. C., Hederich, C., \& García, A. (2015b). Relación entre burnout y engagement académicos con variables sociodemográficas y académicas. Psicología Desde el Caribe, 32(2), 254-267.

Causo-Holgado, M. J., Cuesta-Vargas, A. I., Moreno-Morales, N., Labajos-Manzanares, M. Y., Barón-López, F.J., \& Vega-Cuesta, M. 
(2013). The association between academic engagement and achievement in health sciences students. BMC Medical Education, 13(33), 1-7.

Cavazos, J., \& Encinas, F. C. (2016). Influencia del engagement académico en la lealtad de estudiantes de posgrado: Un abordaje a través de un modelo de ecuaciones estructurales. Estudios Gerenciales, 32, 228-238. https://doi.org/10.1016/j.estger.2016.07.001

Chun-tat, T., \& Siu-man, N. (2012). Measuring engagement at work: Validation of the Chinese version of the Utrecht Work Engagement Scale. International Journal of Behavioral Medicine, 19, 391-297.

D'Mello, S., Dieterle, E., \& Duckworth, A. (2017). Advanced, Analytic, Automated (AAA) measurement of engagement during learning. Educational Psychologist, 5(2), 104123.

https://doi.org/10.1080/00461520.2017.1281747

Domínguez, J., López-Castedo, A., \& Iglesias, E. (2017). Prevalencia del síndrome de burnout en personal de centros de menores: Diferencias según su contexto sociolaboral. Acta Colombiana de Psicología, 20(2), 138147.

https://doi.org/10.14718/ACP.2017.20.2.7

Frederiks, J. A., Blumenfeld, P. C., Friedel, J., \& Paris, A. (2005). School engagement. In KA. Moore, \& L. Lippman (Eds.), Conceptualizing and measuring indicators of positive development: What do children need to flourish? (pp. 305-321). Kluwer Academic/Plenum Press.

Granero-Gallegos, A., \& Baena-Extremera, A. (2016). Validación española de la versión corta del Physical Education Classroom Instrument para la medición de conductas disruptivas en alumnado de secundaria. Cuadernos de Psicología del Deporte, 16(2), 89-98.

Guido, P., Mújica, A., \& Gutiérrez, R. (2011). Diferencias en el autoconcepto por sexo en la adolescencia: Construcción y validación de un instrumento. LIBERABIT, 17(2), 139-146.

Instituto Nacional de Estadística (2019). Abandono temprano de la educaciónformación de personas de 18 a 24 años. España y UE-28. España: INE.
Korobova, N., \& Starobin, S. S. (2015). A comparative study of student engagement, satisfaction and academic success among international and American students. Journal of International Students, 5, 72-85.

Kuh, G. D. (2000). The NSSE 2000 report: National benchmarks for effective educational practice. Center for Postsecondary Research and Planning.

LaNasa, S. M., Cabrera, A. F., Trangsrud, H. (2010). The construct validity of student engagement: A confirmatory factor analysis approach. Res. High Education, 50, 315-322.

Lara, L., Domínguez-Lara, S., Gómez-Espino, J. M., Avecedo, F., Aparicio, J., Saracostti, M., \& Miranda-Zapata, E. (2021). Adaptación y validación del Cuestionario de Compromiso Escolar en países Iberoamericanos. Revista Iberoamericana de Diagnóstico y Evaluación - e Avaliação Psicológica, 59(2), 95-108. https://doi.org/10.21865/RIDEP59.2.08

Lara, L., Saracostti, M., Navarro, J. J., de Toro, X., Miranda, E., Marie, J., \& Fuster, J. (2018). Compromiso escolar: Desarrollo y validación de un instrumento. Revista Mexicana de Psicología, 35(1), 52-62.

Luo, Z., Dang, Y., \& Xu, W. (2019). Academic Interest Scale for Adolescents: Development, validation and measurement invariance with Chinese students. Frontiers, 10, 1-14. https://doi.org/10.3389/fpsyg2019.02301

Maluenda, J., Varas, M., Díaz, A., \& Bernardo, A.B. (2020). Propiedades Psicométricas del University Student Engagement Inventory en Estudiantes de Ingeniería Chilenos. Revista Iberoamericana de Diagnóstico y Evaluación - e Avaliação Psicológica, 57(4), 77-90.

Mañas-Rodríguez, M. M., Alcaraz-Pardo, L., Pecino-Medina, V., \& Limbert, C. (2016). Validation of the Spanish Version of Soane's ISA Engagement Scale. Journal of Work and Organizational Psychology, 32, 87-93.

Martos, Á., Pérez-Fuentes, M. C., Molero, M. M., Gázquez, J. J., Simón, M. M., \& Barragán, A. (2018). Burnout y engagement en estudiantes de ciencias de la salud. European Journal of Investigation in Health, Psychology and Education, 8(1), 23-36.

Medrano, L. A., Moretti, L., \& Ortiz, A. (2014). Medición del engagement académico en 
estudiantes universitarios. Revista Iberoamericana de Diagnóstico y Evaluación - e Avaliação Psicológica, 40(2), 114-123.

Mills, M., Culbertson, S., \& Fullagar, C. (2012). Conceptualizing and measuring engagement: An analysis of the Utrecht Work Engagement Scale. Journal Hapiness Study, 13, 519-545.

Miranda-Zapata, E., Lara, L., Navarro, J.J., Saracostti, M., \& de-Toro, N. (2018). Modelización del efecto del compromise escolar sobre la asistencia a clases y el rendimiento escolar. Revista de Psicodidáctica, 1-9. https://doi.org/10.1016/j.psicod.2018.02.003

Moher, D., Shamseer, L., Clarke, M., Ghersi, D., Liberati, A., Petticrew, M., ... PRISMA-P Group. (2015). Preferred reporting items for systematic review and meta-analysis protocols (PRISMA-P) 2015 statement. Systematic Reviews, 4(1), 1-9.

Navarro, E., Expósito, E., López, E., \& Thoilliez, B. (2014). EPIBI: Escala de Percepción de Indicadores de Bienestar Infantil. Validación del instrumento utilizando modelos politómicos de Rasch. Revista de Educación, 364, 39-65.

Peña, G., Cañoto, Y., \& Angelucci, L. (2017). Involucramiento académico: Una escala. Revista Páginas de Educación, 10(1), 114136. https://doi.org/10.22235/pe.v10i1.1361

Pérez-Fuentes, M. C., Molero, M. M., Barragán, A. B., Martos, Á., Simón, M. M., \& Gázquez, J. J. (2018). Autoeficacia y engagement en estudiantes de ciencias de la salud y su relación con la autoestima. Publicaciones, 48(1), 193-210.

Pérez-Fuentes, M. C., Molero, M. M., Simón, M. M., Oropesa, N. F., \& Gázquez, J. J. (2020). Validation of the Maslach Burnout InventoryStudent SURVEY IN Spanish adolescents. Psicothema, 32(3), 444-451. https://doi.org/10.7334/psicothema2019.373

Ramos-Díaz, E., \& Rodríguez-Fernández, A. (2016). Validation of the Spanish Version of the School Engagement Measure (SEM). The Spanish Journal of Psychology, 19, e86, 1-9.

Renshaw, T. L., \& Long, A. C. J. (2015). Assesing adolescents' positive psychological functioning at school: Development and validation of the student subjective wellbeing questionnaire. School Psychology Quartely, 30(4), 534-552.

https://doi.org/10.1037/spq0000088

Sánchez, M. E., \& Andrae, P. (2020). Análisis factorial confirmatorio de la Escala de Involucramiento Escolar para Adolescentes (EIE-A). Revista Actualidades Investigativas en Educación, 20(1), 1-18.

https://doi.org/10.15517/aie.v20i1.40020

Sánchez-Sandoval, Y., \& Verdugo, L. (2016). Desarrollo y validación de la Escala de Expectativas de Futuro en la Adolescencia (EEFA). Anales de Psicología, 32(2), 545554.

https://doi.org/10.6018/analesps.32.2.205661

Schaufeli, W., Bakker, A., \& Salanova, M. (2006). The measurement of work engagement with a short questionnaire. A cross-national study. Education and Psychological Measurement, 66(4), 701-716. https://doi.org/10.1177/001316440528247

Schaufeli, W., Salanova, M., González-Romá, V., \& Bakker, A. (2002). The measurement of engagement and burnout: A two simple confirmatory factor analytic approach. Journal of Happiness Studies, 3(1), 71-92. https://doi.org/10.1023/a:1015630930326

Schaufeli, W., \& Bakker, A. (2004). Utrecht Work Engagement Scale: Preliminary manual. Utrecht University.

Schaufeli, W. B., Salanova, M., González-Romá, V., \& Bakker, A.B. (2002). The measurement of engagement and burnout: A confirmative analytic approach. Journal of Happiness Studies, 3, 71-92.

Sinatra, G. M., Heddy, B. C., \& Lombardi, D. (2015). The challenges of defining and measuring student engagement in science. Educational Psychologist, 50(1), 1-13. https://doi.org/10.1080/00461520.2014.10029 24

Soane, E., Truss, C., Alfes, K., Shantz, A., Rees, C., \& Gatenby, M. (2012). Development and application of a new measure of employee engagement: The ISA Engagement Scale. Human Resource Development International, 15(5), 529-547.

Tomás, J. M., Gutiérrez, M., Sancho, P., Chireac, S. M., \& Romero, I. (2016). El compromiso escolar (school engagement) de los 
adolescentes: Medida de sus dimensiones. Enseñanza \& Teaching, 34(1), 119-135.

Veiga, F. (2012). Proporsal to the PISA of a new scale of Student's engagement in school. Procedia, Social and Behavioural Sciences, 46, 1224-1231.

Veiga, F. H. (2013). Envolvimento dos alunos na escola: Elaboração de uma nova escala de avaliação. International Journal of Developmental and Educational Psychology, l(1), 441-450.

Veiga, F. H. (2016). Assessing student Engagement in School: Development and validation of a four-dimensional scale. Porcedia, Social and Behavioral Science, 217, 813-819.

Wang, M. T., \& Fredricks, J.A. (2014). The reciprocal links between school engagement, youth problem behaviors and school dropout during adolescence. Child Development, 85(2), 722-737.

Whitney, B. M., Cheng, Y., Brodersen, A. S., \& Hong, M. R. (2019). The scale of student engagement in statistics: Development and initial validation. Journal of Psychoeducational Assessment, 37(5), 553-565. https://doi.org/10.11770734282918769983

Zecca, G., Györkös, C., Becker, J., Massoudi, K., de Bruin, G. P., \& Rossier, J. (2015). Validation of the French Utrecht Work Engagement Scale and its relationship with personality traits and impulsivity. Revue Européenne de Psychologie Appliquée, 65, 19-28.

Scott Carter, J., \& Mamadi Corra (2016) Racial resentment and attitudes toward the use of force by police: An over-time trend analysis. Sociological Inquiry. 20(10) 1-20. https://doi.org/10.1111/soin.12136

Torío-López, S., Peña-Calvo, J. V., RodríguezMenéndez, M. C., Fernández García, C. M., Molina Martín, S., Hernández García, J., \& Inda Caro, M. M. (2013). Construir lo cotidiano: Programa de educación parental. Barcelona: Editorial Octaedro.

Vallejo, A., \& Mazadiego, T. (2006). Familia y rendimiento académico. Revista de Educación y Desarrollo - Universidad Veracruzana, 11. Julio-septiembre 